\title{
Clinical Clues to Diagnose Amyloidosis
}

Ronaldo Altenburg Gismondi, Fernanda Costa Azevedo, Larissa Baldassim Lopes, Alec Morse Blanz, Aline Oreiro da Silveira Pacheco, Luiz Felipe Costa Faria, William Shinji Nobre Soussume, Ana Carolina Benchimol Barbosa, Bianca Bastos Xavier Nunes e Silva, Luis Otavio Mocarzel

Departamento de Medicina Clínica, Hospital Universitário Antônio Pedro, Universidade Federal Fluminense, Rua Marques do Parana, 303/ MMC - Niterói - RJ -24033-900, Brazil.

\section{Corresponding Author:}

Dr. Ronaldo Altenburg Gismondi

Email: ronaldogismondi@id.uff.br

This is an Open Access article distributed under the terms of the Creative Commons Attribution License (creativecommons.org/ licenses/by/3.0).

Received Accepted

Published

March 4, 2019

October 19, 2019

December 20, 2019

\begin{abstract}
Background: Amyloidosis is a rare systemic disease characterized by the deposition of an insoluble protein, amyloid, in various tissues and organs. The disease is more prevalent in the elderly and can affect any organ, including the kidneys, heart, muscles or skin. Furthermore, clinical manifestations may be easily identified during a physical examination and serve as a clue to the diagnosing amyloidosis as well as its etiology. Case Report: The present paper describes the case of an elderly man presenting with macroglossia, "shoulder pad" sign and periorbital ecchymosis. A presumptive diagnosis of amyloidosis in its primary form was made by the attending physician. Afterwards, bone marrow biopsy confirmed multiple myeloma as the underlying cause and the patient was treated with chemotherapy. Conclusion: Macroglossia, "shoulder pad" and periorbital ecchymosis are important physical examination clues to diagnosis and etiology of amyloidosis.
\end{abstract}

Keywords: Amyloidosis, Ecchymosis, Multiple Myeloma, Macroglossia, Physical Examination.

\section{Introduction}

Amyloidosis is a rare systemic disease characterized by the deposition of an insoluble protein, called amyloid in previously healthy tissue and organs [1]. The characteristic manifestations of the disease can be easily identified during the physical examination and can serve as the basis for a more thorough investigation and treatment [2,3]. Macroglossia is a classic presenting sign of amyloidosis, although it is more common in the light chain form of amyloidosis [4]. Rarely, amyloidosis is localized to only this region and, as a result, is associated with better outcomes [4]. Pseudohypertrophy of the shoulder muscles, although typical of amyloidosis, is a rare symptom and occurs as a result of the amyloid deposition near the glenohumeral articulation. This pseudohypertrophy is known as the "shoulder pad" sign, since it is visually similar to the equipment worn on the shoulders by American football players [5]. The cutaneous manifestations of amyloidosis occur in approximately $40 \%$ of cases and are clinically very useful since they indicate the presence of a malignant hematologic disease associated with amyloidosis [3].

This case presentation aims to present an elderly patient with macroglossia, the "shoulder pad" sign and periorbital ecchymosis under physical examination. With these signs, the clinician was able to make the syndromic diagnosis of AL type amyloidosis even before the definitive results of the complementary exams.

\section{Case Report}

Patient is a 63-year-old Afro-Brazilian male with chronic hypertension, in use of losartan and amlodipine, who went to the family health doctor of 
his region for dysphagia. During the physical exam, the patient presented with macroglossia and was referred to the Antonio Pedro University Hospital for further investigation. On the first consultation with the patient in our institution, during general inspection, macroglossia, periorbital ecchymosis and "shoulder pad" sign were noticed, leading to the clinical suspicion of amyloidosis [Fig.1,2]. The patient presented with hyperemia along the nasolabial sulcus and an infiltrated aspect along the hard palate during oroscopy.

Lab exams showed anemia (hemoglobin 8.4 $\mathrm{g} / \mathrm{dL}$ ), hypercalcemia and proteinuria. Later exams determined the presence of an IgG $\lambda$ monoclonal peak during the electrophoresis of serum proteins. There was also a rise in beta-2-microglobulin serum levels and an X-ray showed lytic lesions in the cranium. Echocardiography and cardiac magnetic resonance imaging (MRI) were normal. A shoulder MRI showed signs of amyloid infiltration in the musculoskeletal tissue. A biopsy of the bone marrow demonstrated multiple myeloma. A biopsy of the tongue was also performed and demonstrated the presence of amyloid deposits using the Congo Red stain and polarized light. The patient began the appropriate chemotherapy, with a remarkable improvement in symptoms. The patient is presently under outpatient supervision with remission of symptoms.

\section{Discussion}

Primary amyloidosis can present as different clinical syndromes, challenging the diagnosis. The renal form of the disease is the most common and generally manifests itself as nephrotic syndrome. The heart is the second most affected organ, frequently resulting in restrictive cardiomyopathy, presenting as diastolic cardiac insufficiency syndrome. Nevertheless, as occurred in the present case, the alterations during general inspection can be the initial clinical presentation and their prompt recognition can form important clues to the diagnosis of amyloidosis.

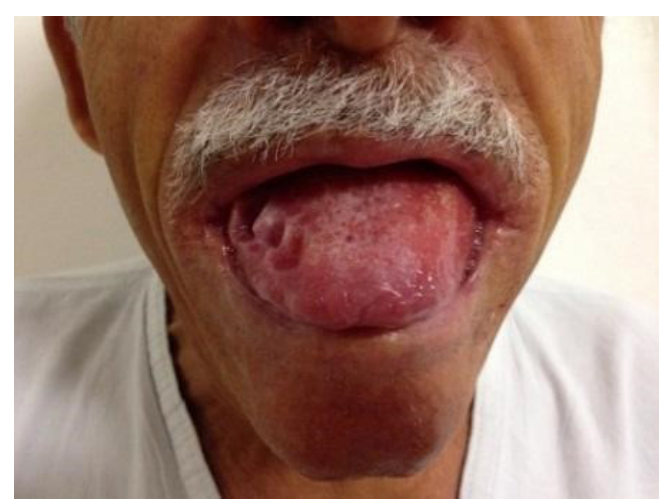

Fig.1: Macroglossia in reported patient.

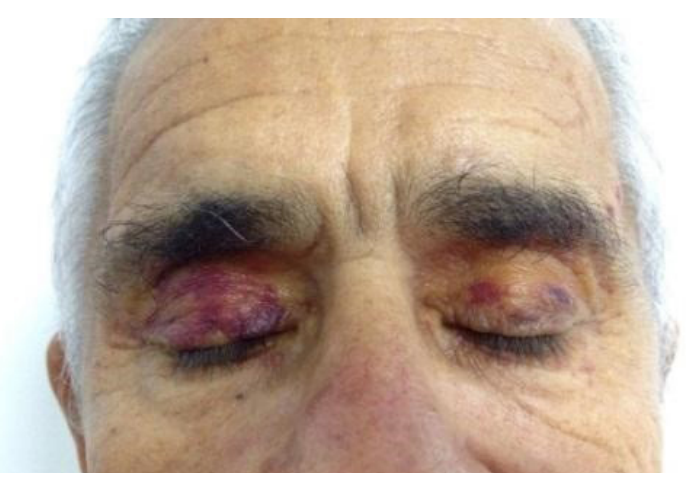

Fig.2: Periorbital ecchymosis in reported patient.

Musculoskeletal syndromes are relatively rare, occurring in about $7 \%$ of cases, but can be the primary clinical manifestation, identified either by the patient themselves or by the attending physician $[5,6]$. These manifestations may include pseudo-hypertrophy of the skeletal muscles, macroglossia, dysphagia and/or palpable nodules located within muscles [6]. Macroglossia is one of the most common musculoskeletal manifestations and occurs in upto $19 \%$ of individuals with $\mathrm{AL}$ type amyloidosis [1,7]. The deposits may lead to indentations, generalized increase in tongue volume, yellow nodules or elevated lesions with white color, especially along the lateral borders. Dysarthria, dysphagia, and obstructive respiratory insufficiency are the principal complications associated with the disease. In the present case, the patient presented with dysphagia, which had been the original reason for the medical consultation and was the first clue in the clinical investigation. 
Amyloidosis related myopathy is commonly associated with the primary (AL) type of systemic amyloidosis, with multiple myeloma being the most frequent underlying etiology $[1,2,5,6]$. The most frequent clinical manifestations are an increase in muscle volume (pseudohypertrophy) due to the amyloid infiltration, muscle weakness (paresis), arthropathy and osteopathy $[6,8]$. The clinical presentations can simulate rheumatological diseases such as rheumatoid arthritis and rheumatic polymyalgia. The patientherein described presented with an increase in shoulder width, known as the "shoulder pad" sign, although he experienced neither pain nor functional limitations of the articulation. An MRI showed amyloid infiltration of the muscular tissue without any intra-articular damage.

The musculocutaneous form of amyloidosis occurs in approximately $40 \%$ of cases and can serve as a clue to plasma cell dyscrasia as the underlying cause of amyloidosis $[1,9,10]$. Among the cutaneous manifestations, periorbital ecchymosis, also known as the "racoon" sign, is the manifestation most commonly associated with amyloidosis [10]. Another form of cutaneous lesion is purpuric macules along the nasogenian sulcus, cervical and axillary regions, which result from capillary fragility due to the deposition of amyloid fibrils along the vessel walls. Yellow or hemorrhagic papules or plaques may occur along skin folds or the oral mucosa, especially on the tongue. Less common dermatologic manifestations include nail dystrophy, infiltrative plaques along the extremities and verrucous lesions on the genitals [10]. The patient in this case presentation presented with the "racoon" sign and with purpuric macules on the torso and left upper arm.

\section{Conclusion}

Amyloidosis is a rare condition with a higher prevalence among the elderly. The diagnosis is confirmed with a histopathologic exam, but a suspicion of the diagnosis is eminently clinical. Therefore, the clinician should be attentive to the principal syndromes associated with amyloidosis.
The patient described presented with classic signs on physical examination and the knowledge of the relation between the clinical manifestations and the types of amyloidosis helped define the next steps of the diagnostic investigation. It is important to familiarize and instruct primary care physicians to recognize the clinical manifestations related to amyloidosis in order to orient the correct investigation and referrals within the health care network.

Contributors: RAG, FCA: manuscript writing, and literature review and references; LBL, AMB, AOSP: critical inputs into the manuscript and patient management; LFCF, WSNS, ACBB, BBXNS, LOM: literature review and discussion. RAG will act as a study guarantor. All authors approved the final version of this manuscript and are responsible for all aspects of the study.

Funding: None; Competing interests: None stated.

\section{References}

1. Kyle RA, Bayrd ED. Amyloidosis: review of 236 cases, Medicine (Baltimore). 1975;54(4):271-299.

2. Pinney JH, Smith CJ, Taube JB, Lachmann HJ, Venner CP, Gibbs SDJ, et al. Systemic amyloidosis in England: an epidemiological study. Br J Haematol. 2013;161(4):525532.

3. Obici L, Perfetti V, Palladini G, Moratti R, Merlini G. Clinical aspects of systemic amyloid diseases. Biochim Biophys Acta. 2005;1753(1):11-22.

4. Fahrner KS, Black CC, Gosselin BJ. Localized amyloidosis of the tongue: a review. Am J Otolaryngol. 25(3):186-189.

5. Elsaman AM, Radwan AR, Akmatov MK, Della Beffa C, Walker A, Mayer CT, et al. Amyloid arthropathy associated with multiple myeloma: a systematic analysis of 101 reported cases. Semin Arthritis Rheum. 2013;43(3):405-412.

6. Gertz MA, Kyle RA. Myopathy in primary systemic amyloidosis. J Neurol Neurosurg Psychiatry. 1996;60(6):655-660.

7. Weiss LS, White JA. Macroglossia: a review. J La State Med Soc. 1990;142(8):13-16.

8. Keith J, Afshar-Ghotli Z, Roussev R, Ernst B, Young B, Bilbao JM. Myopathy as the initial manifestation of primary amyloidosis. Can J Neurol Sci. 2011;38(1):161164.

9. Lestre S, Gonçalves A, João A, Ferreira A, Apetato M. Purpura: primary systemic amyloidosis manifestation. Acta Médica Port. 2009;22(3):307-312.

10. Fernandez-Flores A. Cutaneous amyloidosis: a concept review. Am J Dermatopathol. 2012;34(1):1-14;quiz 15-17. 